\title{
THE REACTIONS OF AMBLYSTOMA TIGRINUM TO OLFACTORY STIMULI ${ }^{1}$
}

\author{
J. S. NICHOLAS \\ Osborn Zoölogical Laboratory, Yale University, New Haven, Connecticut \\ ONE FIGURE \\ INTRODUCTION
}

The existence of the sense of smell in fishes has been clearly shown by Parker, Sheldon, and others. This work has settled the question for this group in accordance with Sherrington's definition, which places the sense of smell in the exteroceptive system and that of taste in the interoceptive system.

Comparatively little work of an exact nature has been done upon the olfactory sense of amphibia. Reese ('12) tested Diemyctilus with food and meat juices, but as he failed to control the sense of sight, his results are not fully conclusive.

Copeland ('13) repeated the experiments of Reese, using more exact methods. He controlled the visual sense by stimulating the olfactory epithelium with diffusion from a motionless source, and he concludes that there is a definite olfactory sense in this animal.

Risser ('14), working upon larvae and adults of the frog and the toad, showed that tadpoles of the toad possess an olfactory sense, while frog tadpoles do not. In the adult toad, he was unable to obtain satisfactory data in regard to the existence of an olfactory sense except in regard to specific odors. $\mathrm{He}$ did not, however, obtain any response to food substances with which the animals normally come in contact.

Burr ('16) obtained definite olfactory responses in larval Amblystoma punctatum.

An investigation was begun in the spring of 1919 in an endeavor to study the correlation of the senses of sight and smell after

${ }^{1}$ Anat. Rec., vol. 20, 1921, p. 189. 
extirpation of the sense organs in the larvae. Unfortunately, all of the animals so treated failed to survive. The present work was started in the fall of 1919 in order to get control results for the data which it is hoped can later be obtained upon adult forms which have been operated upon in early stages of development.

I take pleasure in thanking Dr. Henry Laurens, under whose direction the work has been carried on, for many helpful suggestions and much useful criticism. The general observations upon the behavior of the operated larvae here described were made by Doctor Laurens, who performed the necessary operations in the spring of $1917 .^{2}$

\section{MATERIAL AND METHODS}

The same general method of approach was used as in Burr's ('16) work, i.e., removing the embryonic anlage of the sense organs. Since the method of operation has been described in earlier papers (Laurens, '14, and Burr, '16), no description will be necessary here. The operated larvae were kept in battery jars stocked with the small crustacea, Daphnia and Cypris, and were allowed to grow to a length of about $25 \mathrm{~mm}$. before any of the observations were made.

The adult animals, which were used for the main portion of the work, were three individuals selected from a number that had been sent to Doctor Laurens from Albuquerque, New Mexico, in the spring of 1919. The same individuals were used under all the different conditions described in the experimental section, in order that the general control of the reactions might be more accurate and a more reliable average of reaction obtained.

The methods applied to these forms were mainly those which have been used by Parker in his work on Fundulus and Ameiurus, with an application of some of Risser's methods of experimentation. In all the experiments the test aquarium was cleansed after each operation and filled with fresh water before continuing the tests.

2 The larvae on which the general observations were made were reared from eggs sent to Doctor Laurens by Prof. C. P. Sigerfoos, in the spring of 1917. Dr. W. C. Allee also was kind enough to send the author the eggs of A. tigrinum, but unfortunately, owing to the vicissitudes of the journey and the shock of subsequent operation, these animals did not survive. 


\section{NORMAL BEHAVIOR}

The larvae are in almost incessant motion under laboratory conditions, swimming about the aquaria apparently in a constant search for food. Only after they have become gorged with food will they sink to the bottom, and even then they are seldom motionless, but continue to move about by combined walking and swimming movements, stopping to examine any object that attracts their attention.

The adults used in this work had lived under laboratory conditions for three months previous to the time of the experimental work. The movements of the adult animals are ordinarily not particularly rapid, but the application of stimuli causes a remarkable acceleration of reaction. If placed in an aquarium provided with a float and left undisturbed, the animals will generally be found perfectly motionless upon the float, or else partly submerged with the head protruding above the surface. When placed in an aquarium filled with water to a depth of 6 or 7 inches, but without a float, the animals are restless for a time after their introduction, due probably to the handling which they have received. They soon come to rest, however, with only an occasional sluggish movement. The animals were admirable for the experimental conditions imposed. They can be handled singly and show no trace of the educative process which is said to occur in some forms, viz., Ameiurus, after having been subjected to trials of this nature. An attempt was made to obtain evidence of any difference in reaction which might occur in this way by taking the speed of the reaction as an indicator. The last control experiments, with the conditions as nearly as possible like the original experiment, showed an average time slightly longer than the original.

During the process of acclimatization, the animals were fed solely upon small pieces of earthworm. The degree of visual sensibility is high. In many cases the animals will secure the earthworm while it is sinking to the bottom of the aquarium. If the food has come to rest, the animals often take great interest in the observer, and for this reason the walls of the aquarium 
must be screened for accurate observation in experimental work. The screening has the added advantage of reducing the amount of light in the aquarium and rendering the animals more quiet than they are under a light of higher intensity. The animals have a slight tendency to be more restless than usual under conditions of hunger.

\section{RESPIRATORY MECHANISM}

Bruner ('14a) has described the mechanism of the olfactory sense organ in relation to the respiratory mechanism in a comparative study of a number of Amphibia. He states that in Amblystoma larvae the olfactory process is similar to that of frog tadpoles. "Respiratory water is taken through the nostrils and is the only medium of smell." He finds that the passage from the mouth to the nasal cavity is occluded by a fold of mucous membrane, the choanal valve, which prevents either air or water from passing out in this direction. These choanal valves persist until metamorphosis. He distinguishes in general two distinct types of respiratory. mechanism: in one the respiratory medium is allowed to pass from the nasal cavity to the buccal cavity, the passage of return being occluded by the choanal valves; in the other the respiratory mechanism is completely under muscular control and the respiratory medium can pass freely in and out of the nasal cavity. According to the type of respiratory mechanism present, he classifies the forms as "monosmatic, single smellers, in which the olfactory organ is used to test only the external medium; and diosmatic forms, double smellers, including Siren, Cryptobranchus, Amphiuma, larvae of lungless salamanders and the adult stage of higher amphibians." In the diosmatic forms, the olfactory organ is used in testing the content of the buccal cavity as well as that of the external medium.

Bruner ('14b) mentions that Jacobson's organ is present in Amblystoma adults and that, from the existence of this organ, this form should be classed as a diosmatic form.

As a preliminary to the experiments on the olfactory sense, it was deemed expedient to make a study of the water currents 
passing through the respiratory mechanism and their direction and disposal.

Blinded adult Amblystoma were used on account of the ease with which they can be handled as well as the fact that they show less reaction to the light stimulus occurring under the conditions necessary for observation.

The animals were placed in a flat glass dish under the binocular microscope and the water currents observed by means of a carmine suspension. Three different courses of these currents have been observed. 1) A current taken in through the nasal openings and expelled through the mouth. This condition is the customary one persisting during the state of rest of the animal. 2) A current entering through the nasal passage and the mouth and expelled through the same channels. This is the condition occurring under stimulation, both the incurrent and the excurrent phases taking place with a quick spurt. 3) A current of water expelled through the nasal passages (this being the discharge of the content of the buccal cavity), a special case occurring only when the animals comes to the surface to obtain 'gulps' of air. Air is taken in either through the nose or the mouth. The latter is used for the quick 'gulps' of air which the animal secures as it appears momentarily upon the surface, while the nasal apertures are used when the animal is in a state of rest, lying in the water with the snout protruded above the surface. The current designated above as 3 occurs infrequently as the aftermath of the second method. As a stimulus becomes attenuated by diffusion, the animal seems to take in water more quickly through the mouth that it can be discharged. At such a time the currents seem to be simultaneously going into the mouth and out through the nasal passages. ${ }^{3}$

\footnotetext{
${ }^{3}$ Vincent and Cameron find that respiratory movements cease in the frog as soon as the nares of the animal are under water. This condition has been observed by them over several weeks. A preliminary set of experiments, in which normal, anaesthetized and decerebrate frogs were used, shows that the inhibition of the respiratory movements is temporary and that water currents can be detected by means of the method given above. The efficiency of the valves covering the nares decreases with the time of immersion. None of the animals used in this work survived twenty-four hours of immersion.
} 


\section{EXPERIMENTS ON LARVAE}

Four series of larvae were kept under observation, namely, 1) eyeless, those animals in which the optic vesicles had been removed; 2) noseless, those which had been deprived of the nasal placodes; 3) eyeless and noseless, those from which both the optic vesicle and the nasal placodes had been removed; 4) a series of normal animals which served as controls and were of considerable use in a comparative study.

Under practically the same conditions, it could be easily seen that the normal larvae soon outdistanced the operated in growth. The noseless animals were slightly smaller than the normals of the same age. Next in size were the eyeless forms, and finally the eyeless and noseless, which were considerably smaller than the eyeless.

In a general way, the rate of growth shows the relative importance of the senses of sight and smell. The sense of sight is of more use in the obtaining of food at this stage than is that of smell. If the conditions of the amount of food present are varied, however, there is a change in the relative importance of the senses, for when food is scarce the sense of smell seems to dominate. This is shown in the following observation. The noseless forms seem to have no ability for distinguishing whether a particle is a food substance unless that particle has motion. The eyeless forms have a distinct advantage in this connection for they will distinguish pieces of organic material. If pieces of dead earthworm are placed in an aquarium, the noseless animals will push them around as they do other bits of debris, but will seldom snap at them. The eyeless, in direct contrast to this, will devour the earthworm immediately after coming in contact with it.

A marked difference was noted in the responses to rapidly diffusing food substances obtained in the different groups of the larvae. When freshly cut earthworms are placed in the aquaria, the normal animals begin to swim rapidly about without making any attempt to locate the stimulus. They will often snap as they pass through the area occupied by the diffusing substances, 
but keep swimming on and do not stop at the point of the diffusion which would supposedly be, in this case, the center of attraction. This stimulus is not sufficiently definite and the animal swims about until attracted (by sight) to the motion of the piece of worm, which is then seized.

The same indications are given with beef juice or with strips of beef instead of earthworm. The animals are stimulated by the diffusion, but seldom engulf the food if it remains motionless. When the strip of beef is moved by means of an attached thread, the animal is quickly attracted and speedily engulfs it.

The eyeless forms, under the same conditions, also show signs of stimulation. Their ability to locate food is less than that of the normal larvae. They are easily attracted to any slightly moving object, and since they lack the sense of sight it must be concluded that there is another factor entering into the reaction, that of sensitivity to vibration stimuli. Under conditions of starvation, an increase in sensitivity to the diffusion of passive or motionless objects must result, for the eyeless larvae will locate and devour the fecal material within the aquaria.

The noseless forms are not stimulated by diffusing substances. They swim passively about the aquaria after the introduction of food materials of different sorts and unless the food exhibits motion it is entirely overlooked. Pieces of beef are completely ignored, producing no modification in the behavior of the animal unless they are agitated, when they are quickly engulfed.

These experiments, made on larvae of Amblystoma tigrinum, thus confirm the results of Burr ('16), who used A. punctatum. The work has been further extended by the study of individuals deprived of both eyes and nose.

The eyeless and noseless forms show the same behavior as the noseless. In general, these larvae remain quiet in the aquaria and move about only at intervals. They are absolutely dependent upon mechanical stimuli in finding food. This is clearly shown by the way in which they ignore organic material such as killed earthworm or bits of beef. Under conditions of starvation, they exhibit a certain foraging reaction, pushing the objects about within the aquarium in order to discover whether inherent 
motion is present in these objects. If such motion occurs, the animal is stimulated mechanically. At times, however, these animals will snap at any object with which they may come in contact and engulf it with a subsequent elimination of the substances unfit for food.

In addition to the observations described above, several experiments were made using balls of filter-paper which had either been previously treated with juices of beef or earthworm or had been left untreated. A few experiments with sacks containing test substances were also made. As the result of these tests it may be said that there is a strong indication that the eyeless larvae distinguish their food substances by the sense of smell and mechanical stimulation. The normal animals are mainly dependent upon sight for the final location of food. The noseless larvae are dependent upon visible motion. The noseless and eyeless larvae have only mechanical stimuli to guide them.

EXPERIMENTS UPON THE ADULTS

\section{The reactions of normal animals in the light}

Reactions to motionless objects. The method used in this case was the same as that used by Parker and Sheldon in their experiments with Ameiurus, Mustelus, and Fundulus.

An individual was placed in an aquarium, the sides of which had been screened to obviate any distractions that might occur from the presence of the observer. The aquarium was a glassenclosed one, $42 \mathrm{~cm}$. in length and $26 \mathrm{~cm}$. wide. This was filled to a depth of $10 \mathrm{~cm}$. with water. After allowing a period of ten minutes for the animal to become adapted to the test aquarium, small cheesecloth bags, equal in size and suspended at the same height in the water, were introduced. These bags allowed a rapid diffusion of any substances that might be contained within them. The bags were hung from a rod at the top of the aquarium by means of a cotton thread. The time taken for the introduction of the test materials was at that period at which the animal had come to rest at some position near the central portion of the aquarium. The bags were then introduced with as little disturbance of the medium as possible. Because of the variation of 
the conditions under which experimentation took place, the stimulus caused by the introduction of the test sacks is negligible. One of the bags was filled with minced earthworm and was weighted with sand, while the other bag contained sand alone. Ten trials were made in each series, the three animals being tested in turn with at least a thirty-minute interval between trials.

Under normal conditions, the adult Amblystoma moves slowly about the aquarium seeking for a projection upon which it can rest, preferably upon the surface of the water. Under conditions of stimulation the animal becomes restless, hastening its ordinary movements and proceeding with a greater degree of rapidity about the aquarium. It is this increase in activity that is spoken of in the following series as the 'beginning reaction.' All reactions in this series were considered to be consummated when the testing material was seized by the animal.

The conditions under which each animal was tested were constant in regard to the motion of the test substances. The other factors, however, such as the distance of the test substance from the animal, the distance of the test substances from each other, and the position of the test substances with reference to the animal were considered. The variations in the conditions preclude any factor that might arise in connection with the above.

The response to the stimulus was, in every case, a positive one. Differentiation between the test substances was always in favor of that sack containing the food material. In order to show a few of the experiments and the variations imposed as well as the procedure, two of the individual cases are given below.

Trial 4. Animal A. Placed in aquarium at 11.05. Animal is restless. Test sacks introduced at 11.20. The animal moves restlessly about sides of aquarium, moving to center of the aquarium where the sacks are suspended within 2 inches of each other, noses earthworm bag, circles sand bag, goes to earthworm bag, noses, snaps, and seizes. Reaction is completed in two minutes.

Trial 7. Animal A. Placed in aquarium at 3.10. Test sacks introduced 3.15. The animal swims about, passes sand bag, 
approaches earthworm bag, and seizes. The animal was detached from bag as quickly as possible and sand bag substituted for it. Animal pays no attention to this bag after nosing it. Meanwhile earthworm bag had been placed at far end of aquarium. Animal turns quickly after nosing sand bag, swims to far end of aquarium, noses bag and seizes. The first reaction is complete in three minutes, the second, seven minutes after the introduction of the sacks.

The results of this series are tabulated in table 1, series 1 (a), at the end of this section.

Reactions to moving objects. The same kinds of testing sacks were used, the conditions of the trials being duplicated in so far as possible with but a single variation, that of a moving object. A rod, from which the testing sacks were suspended, was held by the observer. The time element is partially lost in this case, and because of the varying distances of stimulation due to the motion of the test sacks, it is deemed an unimportant factor in this connection. It is included in the description of the individual trials, in order to show the difference in the complete action time for the trials under consideration. The reaction is considered positive when the test sack is seized or an attempt is made to seize it.

The record of one individual case will be given here as typical of the series. The results are tabulated in table 1 , series 1 (b).

Trial 1. Animal C. Placed in aquarium at 10.50. Sand bag, introduced at 11.00, kept in motion in front of the animal, but held at short intervals at different parts of the body. Animal turns at the first approach of the bag and noses it, after which it paid no attention to it, although it was kept moving about the animal for five minutes. Earthworm bag was substituted for sand bag after sand bag has been drawn to end of aquarium away from animal. Earthworm bag played before animal. Animal follows bag about aquarium, noses it when it comes to rest and then seizes. Sand bag substituted and animal allowed to nose it, does not follow it, but moves away, coming to rest at opposite side of aquarium. 
All the experiments were positive in this series, the animal showing a distinct discrimination between the test substances.

\section{Terrarium experiments}

Reactions to motionless objects. A few experiments were made with the animals in a terrarium. The same substances were used as in the preceding trials. The animals are almost insensible to the presence of test substances, and in but one trial in ten was there any indication of a detection of the difference between the substances contained in the bags. This was the only reaction given, all other trials giving no reaction to either bag. The procedure followed was simply to place the bags near each other at the bottom of the terrarium and await the reaction. The one case recorded as positive showed a reaction about fifteen minutes after the introduction of the materials, the animal nosing the earthworm bag after passing the sand bag, and snapping the earthworm bag.

A comparison between the sensitivity of the adult animal in the air and in the water can easily be obtained in the ordinary process of feeding. Cut earthworm can be held against the nasal apertures of the animal and elicit no response. If, however, the animal is so situated that its nasal openings are immersed in water, a very small diffusion from the forceps in which earthworms have been held is sufficient to give an extremely active response.

Reactions to moving objects. If the test sacks are moved about in a terrarium in the same fashion as that described above for the animals in the water, a decided reaction is obtained. In many cases the animal seizes the bag with such force that it can be lifted from the bottom. This is particularly true with reference to the earthworm bag, for the animal will quickly relinquish the sand bag. It is probable that the basis of discrimination in this case is one of taste. It is likely that this is the same reaction as that obtained in the noseless larvae, when the sense of taste or perhaps of touch, must enter into the reaction of the rejection of particles unfit for food. 
3. The reactions of normal animals in the dark room. (In aquarium)

The animals were dark adapted for two days before the experiments were undertaken and were given no food for three days. After dark adaptation, the animals are noticeably more sensitive to handling and the time required to come to rest is lengthened appreciably. A dim red light of minimum intensity, just sufficient for the observer, after a dark adaptation of forty minutes, to see the animal in profile, was used for the observations. The intensity was so weak that the figures upon a watch dial could be read with difficulty just in front of the screened light source. This degree of light is probably below the visual threshold of Amblystoma (Arey, '19), as evidenced in the reactions of the animals during the period of observation preceding the actual experiments. Ordinarily, with one side of the aquarium more brightly illuminated than the other, the animal, particularly if hungry, will tend to move about the sides of the aquarium with a preference for the brighter side, being often attracted to its own reflection in the glass wall, as well as by slight visible movements at the side of the aquarium. Under the dark condition, the animal moves slowly up and down until it is stopped by the aquarium wall, then turning, it may proceed down the center of the aquarium, showing no particular preference for the wall.

Reactions to motionless objects. The test bags were used as in the preceding section and the description of one individual case will show the procedure with the unvarying positive result (table 1, 3a).

Trial 1. Animal B. Tested by the offering of the two test sacks. The earth worm bag and the sand bag were placed at opposite ends of the aquarium. The animal shows the characteristic motor reaction soon after the introduction of the material, but seems unable to locate it. Finally locates the object. Positions of bags reversed and operation repeated. Animal swims restlessly about for some time before approaching object, noses it, and seizes it. As an additional test, a glass vial containing freshly cut earthworms was placed in the aquarium. The animal immediately becomes restless and, after swimming about for 
short while, makes for the glass tube, approaching it from closed end which was purposely placed toward center of aquarium while open end of tube was situated toward side of aquarium and touching it. After nosing tube, animal follows up side of tube to get to open end, pushing tube across aquarium in an effort to obtain diffusing contents. An empty vial produced no reaction.

Throughout this series of experiments, the reaction to the vial filled with earthworms was much faster than that given to the test sacks. The amount of diffusion is greater in the vial experiments and the diffusion takes place from one side of a definite focus in contrast to the test sacks which diffuse on all sides. It is likely that the strength or concentration of the diffusing substances plays a large part in the difference of the speed of the two reactions.

Reactions to moving objects. The animals were subjected to tests with moving objects as in the preceding section. One typical case will suffice to show the manipulation used and the general result.

Trial 1. Animal B. Sand bag twitched gently in front of animal. Animal follows bag as it is drawn across the aquarium. Animal noses bag when it comes to rest. No further reaction. Trial repeated with same result. Earthworm bag twitched before animal. Response immediate, animal follows, noses when at rest and seizes.

The reaction time of normal animals in darkness is much slower than the reaction time in the light, but none the less definite. The sensitivity of the animal to vibration stimuli seems to be increased, as shown by the fact that the animal will follow the sand bag although moved very gently so that the amount of motion given to the surrounding medium is quite small.

\section{Experiments upon nose-stopped animals}

Many methods for occluding the sense of smell have been tried by experimenters. The closure of the external nares by stitching has been used by Parker and his coworkers. The cotton-wool plug used by Sheldon and later by Parker has been 
satisfactory on the forms used by them. In some cases the olfactory tracts have been cut. The latter method is the most effective, but has the one disadvantage of not permitting a return to the normal for control experiments. The cotton-wool plug has been found to have certain irritating effects upon the nasal epithelium and for this reason as well as for ease of handling, a collodion mask was used for occluding the nasal passages in this work.

The method is quite simple. The animal is removed from the aquarium and wrapped in a small hand towel with only the head projecting. The anterior portion of the head is dried as much as is possible and the collodion applied over the surface prepared in this way and across the nares, making a mask the shape of an inverted $\mathrm{T}$, the base of the $\mathrm{T}$ extending well up between the eyes, while the cross line covers the external nares and extends well around the maxilla. The mixture dries rapidly and during drying causes some irritation. The animal will get rid of the cap if its forefeet are free at this stage. The cap will sometimes be removed by the force of the water from the mouth or by an excess of mucous secretion on the front of the head, but after three or four applications the animal becomes accustomed to the mask and shows little or no sign of irritation. At this stage the nose cap is more or less firmly adherent to the head of the animal. The animal is replaced in the water of the aquarium and generally becomes quiet in ten or fifteen minutes. If the cap has remained fast during this period, it will probably remain fixed for at least twelve hours, during which time experimentation may proceed.

The respiratory process of the animal is changed by this occlusion of the nares. The animal comes to the surface and 'gulps' air by means of the mouth, then sinks and exhales slowly, the bubbles of air escaping at the mouth. The act of respiration affords a constant check upon the effectiveness of the nose mask, for if ineffective the water current under pressure from the buccal cavity will soon remove it. This mask is easily and quickly removed from the animals, after which they respond to control tests in exactly the same fashion as in the normal 
experiments. For the purposes in hand, the method was admirable, although it did require a great deal of patience and considerable observation to make sure that the nose caps were tight. It was with the idea of making a combination hoodwink and nose cap that this method was applied, but the irritation resulting from the application of the mixture of lampblack and collodion to the eyes of the animal was too great to get normal reactions.

Reactions to motionless objects. After securing a nose mask which was satisfactory, the animals were placed in screened aquaria in order that the observer might be concealed from view. The reaction time was much increased. Twelve minutes, three times as long as the average reaction time for the normal, was allowed before a test was pronounced negative. The reactions were recorded as negative when the animal made no effort to obtain either bag and also showed none of the characteristic motor activity of a stimulated animal, as positive when the animal attempted to nose either bag. In the reactions so recorded, this reaction occurred within five to seven minutes after the introduction of test materials into the aquaria. After the attempt to nose, the animal paid no further attention to either of the bags.

The reactions obtained show distinctly that animals with the sense of smell occluded cannot discriminate between test substances, for there was in no case any preference shown, the animals nosing the sand bag, on the average, just as frequently as the earthworm bag. The reaction is, to a large extent, one of contact and is accidental. (Table 1 , series 4 (a).)

Reactions to moving objects. In contrast to the above reactions, the reactions of the nose-stopped animals to moving objects is quite rapid. The test sacks were moved about the aquarium at various distances from the animal. All the reactions gave an average reaction time of two and two-tenths minutes, the animals showing no discriminaton between the test substances, in marked contrast to the selective reaction obtained from animals with the possession of the normal respiratory mechanism. (Table 1 , series $4(\mathrm{~b})$.) 


\section{Experiments with blinded animals}

The animals were anaesthetized with chloretone and the eyes removed with a pair of fine scissors. After several hours the animals seemed normal and were fed. They were then not fed for a period of five days preceding the commencement of the following experiments. Animal A, which was not normal, due to an inflammation of the external nares, was not used in the course of these experiments. The tests were made with the usual test sacks. Results are tabulated in table 1, series 5 (a) and (b).

The average reaction time in this series is almost the same as that for the normal. All the tests showed that the animals discriminated between the test substances, reacting positively to the earthworm sack.

In the reactions to moving objects, ten trials were made, all giving the same end result. The animals were quickly attracted by any movement in the water. They would not, however, follow the sand bag, although stimulation was shown by their motor activity. They did follow the earthworm bag and would, if possible, snap and seize it.

\section{Experiments with nose-stopped and blinded animals}

The external nares of the blinded animals were covered with collodion and the animals were subjected to the same trials as those given above. The results are the same for both the moving and the motionless objects. There was no attraction to either object in the motionless series. In the series with the moving object, there was a slight motor activity present but no definite objective. The results are tabulated in table 1 , series 6 (a) and (b).

\section{\%. Partitioned aquarium experiments}

An aquarium was divided into two compartments by the insertion of a glass partition which ran diagonally across it, having a slit three-eighths of an inch high between the base of the partition and the concrete bottom of the aquarium. This slit afforded the means by which diffusing substances could pass to the test 
side. The arrangement proved very useful in the differentiation of sight stimulus from that of smell and was used on all of the four series of animals. Two kinds of substances were used, diffusible and non-diffusible but visible. For the first, an infusion was made of minced earthworm, generally allowed to stand over night and filtered in the morning. The resulting extract contained no visible solid substance, but simply the juices and body fluids of the earthworms. This proved to be a very potent sort

TABLE 1

Reactions to test sacks

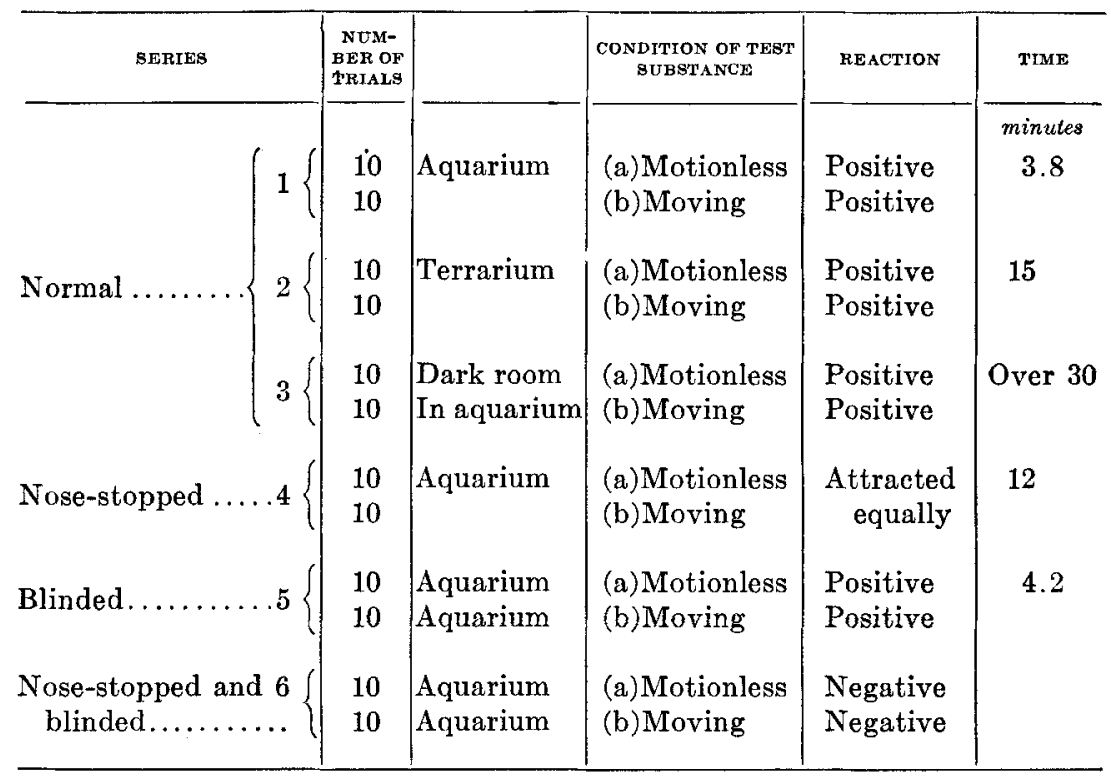

of stimulus. It was applied by means of a pipette along the wall of the aquarium, and diffusing through the water passed under the partition. The observer was screened from view. The reactions were remarkably quick and vigorous, in many cases the animal moved to the central portion of the partition and snapped at the diffusing substances which it perceived but certainly did not see.

The non-diffusing substances used were bits of rubber tubing and uncut earthworms. According to the work of Olmsted ('18), 
uncut earthworms will stimulate Ameiurus by slime secreted by the skin. This is not the case in A. tigrinum, a fact which may indicate that the probable sensitivity of combined air and water breathers is not so great as single smellers. Red rubber tubing of small diameter was used to simulate earthworms. Uncut earthworms were allowed to move about in the half of the aquarium not containing the Amblystoma. The rubber tubing was more effective in eliciting a response if set in motion by means of

TAB́LE 2

Diffusing substances

\begin{tabular}{|c|c|c|c|}
\hline TRIALS & SERIES & SUBSTANCE & REACTIDN \\
\hline 10 & (1) Normal & Earthworm infusion & Positive \\
\hline 10 & (2) Nose-stopped & Earthworm infusion & Negative \\
\hline 10 & (3) Blinded & Earthworm infusion & Positive \\
\hline 10 & (4) Blinded and nose-stopped & Earthworm infusion & Negative \\
\hline \multicolumn{3}{|c|}{ Control, distilled water $\ldots \ldots \ldots \ldots \ldots \ldots \ldots \ldots \ldots \ldots \ldots \ldots$} & Negative \\
\hline
\end{tabular}

TABLE 3

Non-diffusing substances

\begin{tabular}{c|l|l|l}
\hline $\begin{array}{c}\text { NUMBER } \\
\text { OF } \\
\text { TRIaLS }\end{array}$ & \multicolumn{1}{|c|}{ sertes } & strmulus & Reaction \\
\hline 10 & (1) Normal & Uncut earthworm and tubing & Positive \\
10 & (2) Nose-stopped & $\begin{array}{l}\text { Uncut earthworm and tubing } \\
\text { Uncut earthworm and tubing }\end{array}$ & Positive \\
10 & (3) Blinded & Uncut earthworm and tubing & Negative \\
10 & (4) Nose-stopped and blinded & Uncut \\
\hline
\end{tabular}

a cotton thread. Both objects acted solely as visual stimuli, as can be seen from the accompanying table (table 3 ).

\section{Experiments with odor streams}

Risser has described an apparatus with which he tested the olfactory reactions of the toad. The apparatus consisted of a testing chamber into which odors were brought from an odor chamber by means of an air current.

A modification of this method was devised. The source of the air current was a compressed-air system, the air being run 
through a bath of water in a 10-liter container. This container acted as a reservoir and served to keep the pressure constant (fig. 1).

The apparatus was first thoroughly tested for the effects of air currents under various pressures. It was found that an air current evidently stronger than that which Risser used had no effect upon the reactions of the experimental animal. This current could be distinctly felt on the lips or the surface of the tongue of the experimenter.

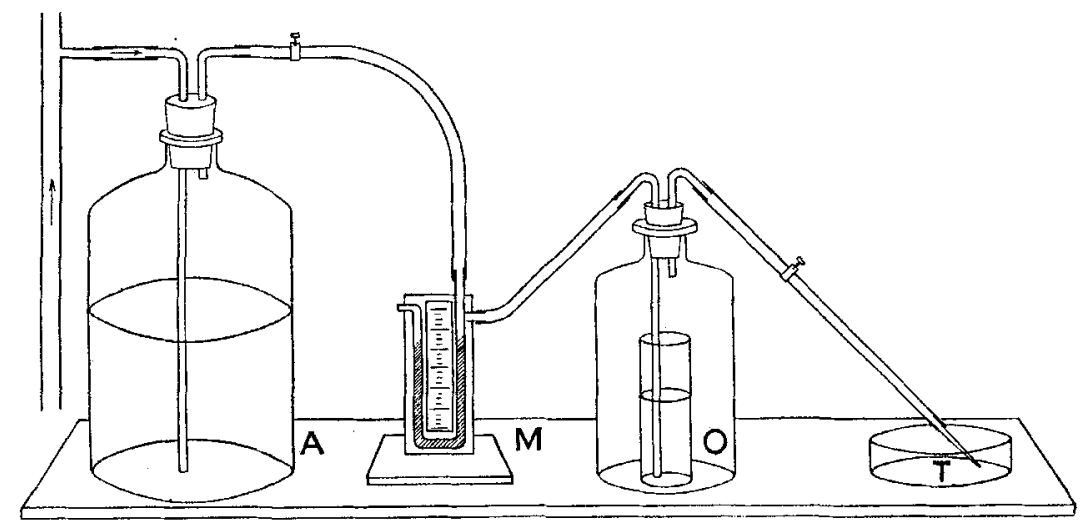

Text-figure 1 Diagram of the arrangement of the odor-stream apparatus From the flask $(A)$ the air was run through a manometer $(M)$, and from there to the odor chamber $(O)$. A fluid, the odor of which was to be tested, was placed in a vial in the odor chamber and the air bubbled through the liquid. A glass nozzle, $0.5 \mathrm{~mm}$. in diameter was used to conduct the odor stream to the testing chamber $(T)$.

For the experiments the Amblystoma were placed in a large dish, the bottom of which was covered with moist sand. Various pressures between 12 and $48 \mathrm{~mm}$. of mercury were tried; temperature, $20^{\circ} \mathrm{C}$. The average was $24 \mathrm{~mm}$. of mercury. Air under this pressure emerging through a $0.5-\mathrm{mm}$. nozzle gives a displacement of $25 \mathrm{cc}$. of water per minute.

One of the individual experiments is here given in order to show the manipulation of the test substances as well as the behavior of the animal under the control trials.

Animal B: Control test with distilled water. Animal placed in testing chamber and allowed to come to rest. Air current 
under pressure of $12 \mathrm{~mm}$. of mercury applied to external nares, the nozzle being held at a distance of $1 \mathrm{~mm}$. from animal. No muscular reaction and no respiratory reaction. Under a pressure of $36 \mathrm{~mm}$., nozzle was held for three minutes with no reaction on part of animal except a raising of head. When air current was directed toward different parts of body (region behind the head, in front of the limb, trunk region, and the tail region) there was no reaction.

If the animals are partly submerged in water and the air current is allowed to produce a series of bubbles, the animal is

TABLE $4^{1}$

Reactions to odor streams

\begin{tabular}{|c|c|c|c|c|}
\hline stimules & $\begin{array}{l}\text { PRES- } \\
\text { SURE }\end{array}$ & $\operatorname{TIMF}$ & REACTION & TRIAL \\
\hline Earthworm. & $\begin{array}{c}m m . \\
24\end{array}$ & & No reaction & \\
\hline Turpentine, oil of. & 24 & $3 \mathrm{~min}$. & No reaction & Negative \\
\hline Carbolic acid. & 24 & $3 \mathrm{~min}$. & No reaction & Negative \\
\hline Oil of cloves. . & 24 & 20 sec. & Shakes head-draws away/I & Positive \\
\hline Oil of wintergreen. & 24 & $3 \mathrm{~min}$. & No reaction & Negative \\
\hline Bergamot.. & 24 & $3 \mathrm{~min}$. & No reaction & Negative \\
\hline Aniline . & 24 & $3 \mathrm{~min}$. & No reaction & Negative \\
\hline Ammonia, concentrated.. & 24 & 1 sec. & Withdraws violently & Positive \\
\hline Formol, 40 per cent....... & 24 & $3 \mathrm{~min}$. & No reaction & Negative \\
\hline Chloroform........ & 24 & $1 \mathrm{~min}$. & Withdraws from current & Positive \\
\hline Distilled water... & 24 & $3 \mathrm{~min}$. & No reaction & Negative \\
\hline
\end{tabular}

1 A trial is designated as negative when there is no reaction af ter three minutes' exposure; positive, when there is motor activity resulting from the stimulus.

mechanically stimulated and snaps at the bubbles. This stimulation is quite strong in the region just anterior to the forelimb, and is active, although less so, in the tail region. The trunk region proper shows but little sensitivity.

In testing odoriferous currents, a maximum exposure of three minutes was allowed, after which the trial was declared to be negative. The reaction was designated as positive if the animal moved toward the air current or away from it. A discussion of the results obtained and a comparison with those of Risser will be found in the next section. 


\section{DISCUSSION}

The observations upon the operated larvae show that $\mathrm{A}$. tigrinum possesses an olfactory sense. They also indicate the relative importance of the senses of sight and smell. Sight is undoubtedly the predominant sense in the obtaining of food, although it is rather interesting to observe that under conditions of paucity of food the animals in which the organ of sight has been removed, long before it could possibly function, have a more highly developed sensitivity toward objects giving an olfactory stimulus than do the normal animals. There seems to be a dominance of one sense over the other, this dominance being clearly detrimental to the animal under adverse conditions. This same condition is demonstrated in the observations upon the adult forms. A comparison between the ability of the blinded adults to obtain food and of normal individuals in the dark room is striking. Blinded adults give a positive reaction to olfactory stimulation in practically the same time as normal animals in the light. Risser has noted that there is what he has termed an inhibitory effect of the eyes over the nose in the toad. It is true that an animal possessing sight cannot accommodate itself to olfactory stimulation under conditions of darkness in the same time that blinded animals which have become accustomed to obtain food substances without the intermediation of sight can do so.

The factor of taste and its relation to the problem must be considered. Although the animals are nose-stopped (tables 1 , series $4 ; 2$, series 2 ; and 3 , series 2 ), the organs of taste are undisturbed by this procedure. Water currents are passing in and out of the animal's mouth and some of the diffusing substance should come in contact with the taste buds. If the taste buds are responsible for the reaction, there should be a reaction even in nose-stopped animals. This is not found. In this case, however, the eye is a considerable factor, as is shown by the reaction elicited by moving objects. In the series with eyes removed and the nasal passages closed (tables 1 , series $6 ; 2$, series 4 ; and 3 , series 4) there is no reaction to any stimulus applied. The sense of taste, therefore, can be regarded as a negligible factor. 
The experiments in the partitioned aquarium (summarized in tables 2 and 3 ) show that animals possessing sight respond to stimuli, which because of their non-diffusibility have no effect upon the olfactory organ. The animals which have been deprived of sight can respond only to diffusing substances. In every case, the animals which could use neither sight nor smell are negative to all forms of olfactory stimulus.

Parker, in his work on fishes, found that the organs of the lateral line and the general chemical sense which are present in the skin had to be occluded in his analysis of results. This was accomplished by painting the animals with magnesium sulphate. This method was tried upon the Amblystoma adult to see if a definite factor of skin sensitivity might be present, but with no result. The use of alkalis or acids such as were employed by Parker and Sheldon ('12) or those used by Crozier ('16) might show the nature of this sense in the larvae of Amblystoma, but in relation to the stimuli employed, the skin sense was not shown to be in any sense discriminatory.

Parker and Sheldon ('12) point out that much of the work done by Aronsohn ('86) in which he uses oil of cloves in order to test the sense of smell in the goldfish, is inconclusive because of the use of an irritating substance. In their own experiments, they used normal substances for testing the olfactory sense.

The results obtained by Risser ('14) with the odor-stream apparatus are inconclusive. He states in his summary: "Odor streams specific in character, made to flow over and into the nasal openings stimulate the olfactory sense organ; such stimulation causing definite motor activities to follow," and further, "appropriate operation are confirmatory that stimulation by such odor streams is olfactory. Section of the olfactory tract inhibits the reactions. Olfactory stimulation and reaction are not affected by section of the ophthalmic branch of the trigeminal nerve."

The materials which Risser used and which, therefore, are to be assumed as odor streams 'specific' in character, are a large number of essential oils together with some food substances. A glance at his data shows that positive reactions were obtained 
with irritating substances. The presence of substances such as ammonia or oil of cloves will cause a motor reaction in A. tigrinum as a reflex of skin irritation.

The results of the present series might be summed up as follows. Normal animals will discriminate between test substances and react positively toward the substance giving an olfactory stimulus, whether that substance is moving or motionless, and whether the animal is under conditions of light or of darkness.

Animals deprived of the olfactory sense by occluding the nasal passages show the same reaction to both test substances and react more quickly to moving substances than do the normal animals. There is no olfactory discrimination.

Animals which have been blinded respond to olfactory stimulus. If a moving object does not give rise to olfactory stimulation, the animal moves about in an indefinite manner without a definite objective, but will locate and try to obtain a moving focus giving an olfactory stimulus.

Animals which have been blinded and nose-stopped give no reactions except to moving objects and show no discrimination between test substances.

Normal animals give no reactions to normal substances carried in odor streams. They will, however, give motor reactions to irritating substances.

\section{SUMMARY}

1. General observations upon the growth and behavior of operated larvae show that the visual sense is the primary sense used in obtaining food.

2. When motionless food substances are the only ones present, the olfactory apparatus functions to a greater extent in eyeless larvae than it does in normal animals.

3. The experiments upon adult animals (table 1) indicate that, while the eye is the most important agent in obtaining food, yet with the nose alone the animals are capable of detecting and locating definite food substances. 
4. When the animals are tested in darkness (table 1, series 3 ), the evident retardation in the time of reaction is due to the dependence of the animal upon the sense of sight.

5. After the removal of the eyes of the animals, they become accustomed to use the olfactory organ only and in this way the remaining sense organ is correlated to the needs of the animal.

6. Experiments performed with diffusing and non-diffusing substances (tables 2 and 3 ) demonstrate the fact that an animal possessing the optic sense is stimulated by non-diffusing substances, while those possessing the olfactory sense are stimulated by diffusing substances.

7. Odor streams involving the use of a number of substances (table 4) elicit no response on the part of the animal unless the substance used possesses irritating properties, in which case the animal responds by a decided motor reaction.

8. Responses to motionless test substances in air (table 1, series 3) show that the animal can find food substances, although the reaction time is longer than if the animal and the test substance are submerged.

9. The experiments indicate clearly that there is a definite olfactory sense in both the larvae and the adults of Amblystoma tigrinum.

\section{LIST OF REFERENCES}

Aronsonn, E. 1886 Experimentelle Untersuchungen zur Physiologie des Geruchs. Arch. f. Physiol, S. 321-357.

Arey, L. B. 1919 A retinal mechanism of efficient vision. Jour. Comp. Neur., vol. 30 , pp. $343-353$.

BRUNER, H. L. 1914 a The mechanism of pulmonary respiration in amphibians with gill clefts. Morph. Jahrb., Bd. 48, S. 63-82.

$1914 \mathrm{~b}$ Jacobson's organ and the respiratory mechanism of amphibians. Ibid., S. 157-165.

BURR, H. S. 1916 The effects of the removal of the nasal pits in Amblystoma embryos. Jour. Exp. Zoöl., vol. 20, pp. 27-49.

Copeland, M. 1912 The olfactory reactions of the puffer or swell-fish, Spheroides maculatus (Black and Schneider) Jour. Exp. Zoöl., vol. 12, pp. $363-368$.

1913 The olfactory reactions of the spotted newt. Jour. Anim. Behavior, vol. 3, pp. 260-273.

Crozigr, W. J. 1916 Regarding the existence of the 'common chemical sense' in vertebrates. Jour. Comp. Neur., vol. 26, pp. 1-8. 
LaURENS, H. 1914 The reactions of normal and eyeless amphibian larvae to light. Jour. Exp. Zoöl., vol. 16, pp. 195-210.

OLMsted, J. M. D. 1918 Experiments on the nature of the sense of smell in the common catfish, Ameiurus nebulosus. Amer. Jour. Physiol., vol. 46, pp. 443-458.

Parker, G. H. 1910 Olfactory reactions in fishes. Jour. Exp. Zoöl., vol. 8, pp. $535-542$.

1911 The olfactory reactions of the common killifish, Fundulus heteroclitus. Jour. Exp. Zoöl., vol. 10, pp. 1-6.

1912 The relation of smell, taste and the common chemical sense in vertebrates. Jour. Acad. Nat. Sci. of Phila., 2nd series, vol. 15, pp. 221-234.

1913 The directive influence of the sense of smell in the dogfish. Bull. U. S. Bur. of Fish., vol. 33, pp. 63-68.

Parker, G. H., and Sheldon, R. E. 1912 The sense of smell in fishes. Ibid., vol. 32 , pp. $35-46$.

Parker, G. H., and van Heusen, Anne P. 1917 The responses of the catfish, Ameiurus nebulosus, to metallic and non-metallic rods. Amer. Jour. Physiol., vol. 44, pp. 405-420.

REESE, A. M. 1912 Food and chemical reactions of the spotted newt. Jour. Anim. Behav., vol. 2, pp. 190-208.

RISSER, JoNATHAN 1914 Olfactory reactions in amphibians. Jour. Exp. Zoöl., vol. 16 , pp. $617-652$.

Sheldon, R. E. 1909 The reactions of the dogfish to chemical stimuli. Jour. Comp. Neur., vol. 19, pp. 273-311.

1911 The sense of smell in selachians. Jour. Exp. Zoöl., vol. 10, pp. $51-62$.

1912 Olfactory tracts and centers in teleosts. Jour. Comp. Neur., vol. 22, pp. 177-339.

Shenrington, C. S. 1906 The integrative action of the nervous system. Scribner's Sons, New York.

Vincent, Swale, and Cameron, A. T. 1920 A note on an inhibitory respiratory reflex in the frog and some other animals. Jour. Comp. Neur., vol, 31, pp. 283-292. 